\title{
(6) OPEN ACCESS \\ Evaluation of seven rapid tests for syphilis available in Brazil using defibrinated plasma panels
}

\author{
Maria Luiza Bazzo, ${ }^{1}$ Leonardo Rapone da Motta, ${ }^{2}$ \\ Renata Cristina Messores Rudolf-Oliveira, ${ }^{1}$ Alisson Bigolin, ${ }^{1}$ Lisléia Golfetto, ${ }^{1}$ \\ Fábio Mesquita, ${ }^{3}$ Adele Schwartz Benzaken, ${ }^{4}$ Pamela Cristina Gaspar, ${ }^{4}$ \\ Ana Flavia Nacif P Coelho Pires, ${ }^{4,5}$ Orlando da Costa Ferreira Júnior, ${ }_{1}^{6}$ Miriam Franchini ${ }^{4}$
}

\begin{abstract}
- Additional material is published online only. To view please visit the journal online (http://dx.doi.org/10.1136/ sextrans-2017-053177)
\end{abstract}

For numbered affiliations see end of article.

\section{Correspondence to} Dr Maria Luiza Bazzo, Laboratório de Biologia Molecular, Microbiologia e Sorologia Departamento de Análises Clínicas. Centro de Ciências da Saúde Universidade Federal de Santa Catarina Campos UniversitárioTrindade Florianopolis, SC, Brazil; m.l.bazzo@ufsc.br, marialuizabazzo@gmail.com

Received 1 March 2017 Revised 13 June 2017 Accepted 27 July 2017

\section{(1) crossuata}

To cite: Bazzo ML, da Motta LR, RudolfOliveira RCM, et al. Sex Transm Infect 2017:93:S46-S50.

\section{ABSTRACT}

Objectives In 2012, the WHO estimated that 6 million new cases of syphilis per year would occur worldwide, including 937000 in Brazil. Early diagnosis and treatment of syphilis are essential to reduce morbidity and prevent transmission. The availability of rapid tests (RTs) for this diagnosis means that testing can be performed more quickly, as a point-of-care test, even in non-laboratory environments and requires only simple technical training to antibodies detection. The objective of this study was to evaluate the performance and operational aspects of seven commercially available RTs for syphilis in Brazil.

Methods Seven rapid treponemal tests were evaluated for sensitivity, specificity, accuracy and Kappa value, according to a panel composed of 493 members.

The operational performance of the assay was also determined for these tests.

Results The seven RTs showed sensitivity ranging from $94.5 \%$ to $100 \%$ when compared with the reference tests and specificity of between $91.5 \%$ and $100 \%$. All the RTs evaluated presented good operational performance, and only one failed to present the minimum specificity as defined by Brazil's Ministry of Health.

Conclusion All the tests presented good operational performance, and the professionals who performed them considered them to be easy to use and interpret. This evaluation is important for making informed choices of tests to be used in the Brazilian Unified Health System.

\section{INTRODUCTION}

Syphilis constitutes a major public health problem. In 2012, the WHO estimated that six million new cases of syphilis per year would occur worldwide. ${ }^{1}$ Between 2014 and 2015, Brazil experienced an increase of $32.7 \%$ in acquired syphilis and $21.0 \%$ in congenital syphilis. ${ }^{2}$

Early diagnosis and treatment of syphilis are essential to reduce morbidity and prevent vertical transmission (congenital syphilis), sexual transmission and further spread of the disease. ${ }^{3}$ However, the complexity of the natural history of syphilis and the absence in many cases of specific clinical manifestations are barriers to the implementation of surveillance programme in developing countries. ${ }^{4}$

Conventional syphilis testing is usually based on serological tests, including antibodies detection by treponemal and non-treponemal tests, performed in laboratories. These tests require the training of laboratory teams. ${ }^{5}$ In contrast, rapid tests (RTs), often referred as point-of-care tests, can be conducted by staff with minimal training at a community setting, rather than sending the sample to a laboratory. The WHO's worldwide policy to eradicate the vertical transmission of syphilis has emphasised the importance of RTs in enabling pregnant women to access diagnosis. ${ }^{3}$ Therefore, the use of treponemal RTs is advocated for screening populations in less developed settings.

The RTs can be used in a variety of situations, such as in difficult-to-reach places, for diagnosing key HIV and indigenous populations and especially for priority testing and assisting pregnant women. The availability of RTs has made it possible for syphilis testing to be done in non-laboratory environments by professionals with no laboratory experience. The objective of this study was to evaluate the performance and operational aspects of seven RTs for syphilis that are commercially available in Brazil.

\section{METHODS}

\section{Sample panel}

The members that comprised the panel used were obtained from bags of plasma that presented reagents and non-reagents for syphilis. These were donated in 2011-2013 by different blood banks in Brazil to the Laboratory of Molecular Biology, Microbiology and Serology (LBMMS) of the Federal University of Santa Catarina.

The selected samples were converted to serum (defibrinated plasma) by adding $1 \mathrm{~mL}$ of thrombin $(100 \mathrm{U} / \mathrm{mL})$ in $1 \mathrm{M}$ calcium chloride for each $100 \mathrm{~mL}$ of plasma, followed by treatment with $10 \%$ (wt/vol) Kaolin, according to Castro et al. ${ }^{6}$

The truly positive (127 samples that were reagent in all reference tests) and truly negative (366 samples that were non-reagent in all reference tests) syphilis plasma samples were fractionated into five aliquots of $350 \mu \mathrm{L}$ each and stored at $-70^{\circ} \mathrm{C}$ in the LBMMS, until the time of the evaluation. Each aliquot was thawed only once. Online supplementary table S1 presents the results of the panel characterisation with treponemal tests and the non-treponemal Venereal Disease Research Laboratory (VDRL) test and the results of the RTs included in the study.

\section{Reference tests}

The reference assays used by the LBMMS were: ARCHITECT System Syphilis TP (Abbott 
Table 1 Characteristics of the seven rapid tests evaluated according to the information provided by the manufacturers

\begin{tabular}{|c|c|c|c|c|c|c|c|}
\hline \multirow[b]{2}{*}{ Test } & \multirow[b]{2}{*}{ Manufacturer } & \multicolumn{3}{|c|}{ Specimen type } & \multirow{2}{*}{$\begin{array}{l}\text { Required } \\
\text { specimen } \\
\text { volume* }\end{array}$} & \multirow[b]{2}{*}{$\begin{array}{l}\text { Storage } \\
\text { conditions }\end{array}$} & \multirow[b]{2}{*}{ Batch used } \\
\hline & & $\begin{array}{l}\text { Whole } \\
\text { blood }\end{array}$ & $\begin{array}{l}\text { Serum or } \\
\text { plasma }\end{array}$ & CSF & & & \\
\hline Determine Syphilis TP & Alere Medical, Matsudo, Chiba, Japan & $\sqrt{ }$ & $\sqrt{ }$ & No & $50 \mu \mathrm{L}$ & $2^{\circ} \mathrm{C}-30^{\circ} \mathrm{C}$ & $61194 k 100$ \\
\hline Imuno-Rápido Syphilis & WAMA Diagnóstica, São Carlos, SP, Brazil & - & $\sqrt{ }$ & No & $50 \mu \mathrm{L}$ & $2^{\circ} \mathrm{C}-30^{\circ} \mathrm{C}$ & $14 C 174$ \\
\hline Rapid Check Syphilis & NDI-UFES, Vitória, ES, Brazil & $\sqrt{ }$ & $\sqrt{ }$ & No & $75 \mu \mathrm{L}$ & $5^{\circ} \mathrm{C}-30^{\circ} \mathrm{C}$ & $\begin{array}{l}\text { SIF131226 } \\
\text { SIF140127 }\end{array}$ \\
\hline RT ABON & Abon, Hangzhou, PR China & $\sqrt{ }$ & $\sqrt{ }$ & No & $50 \mu \mathrm{L}$ & $2^{\circ} \mathrm{C}-30^{\circ} \mathrm{C}$ & SYP4120045 \\
\hline RT DPP Syphilis & Bio-Manguinhos, FIOCRUZ, Rio de Janeiro, RJ, Brazil & $\sqrt{ }$ & $\sqrt{ }$ & No & $10 \mu \mathrm{L}$ & $2^{\circ} \mathrm{C}-30^{\circ} \mathrm{C}$ & 151TY002Z \\
\hline
\end{tabular}

*Volume indicated by the manufacturer for serum samples.

CSF, cerebral spinal fluid.

Laboratories, Wiesbaden, Germany), TPHA100 (bioMérieux, Marcy l'Etoile, France) and Immuno-COM FTA-ABS Syphilis (WAMA Diagnóstica, São Carlos, SP, Brazil). While the Fluorescent Treponemal Antibody Absorption test (FTA-ABS) results were adopted as the gold standard, the results obtained in the Treponema pallidum Hemagglutination assay (TPHA) and the chemiluminescence immunoassay tests were also all coincident with those obtained with the gold standard. The LBMMS participates in the WHO/Centers for Disease Control and Prevention (CDC) Syphilis Serology Proficiency Programme.

\section{Treponemal RTs}

In this study, we evaluated seven commercially available RTs (table 1), which are officially registered by Brazil's National Health Surveillance Agency (ANVISA). Valid ANVISA registration, a mandatory requirement for marketing RTs in Brazil, was a major criterion for inclusion in this evaluation. Tests were performed according to the manufacturers' instructions. The results were read by two independent professionals who were unaware of the results obtained in the reference tests. The kit manufacturers had no participation in the design of the study or the performance of the tests, and only learnt the results from a report disclosed at the end of the evaluation.

\section{Criteria for inclusion in algorithms recommended for use in Brazil}

The Department of STI, HIV/AIDS and Viral Hepatitis (DIAHV) has established the following criteria for the selection and use of RTs on flowcharts recommended for syphilis testing. The test is required to: (A) have a sensitivity of $94.5 \%$ or more; (B) specificity equal to or greater than $93.0 \% 0^{78}$; and $(C)$ an operational performance equal to or greater than 4 points out of a total of 5. ${ }^{910}$

\section{Operational performance}

The analysis of the operational performance of the trial was performed according to criteria previously described with an additional Ministry of Health $(\mathrm{MoH}) /$ Brazil criterion for RTs that should provide a result in less than $30 \mathrm{~min} .{ }^{10}$ A score 0 (negative) or 1 (positive) was assigned to each operational characteristic. To achieve good operational performance RTs had to meet at least four of the following five criteria: storage temperature: (1) ambient temperature, (0) need for refrigeration; number of reagents required to perform the assay: (1) only one reagent, (0) need for more than one reagent; total number of steps to perform the test: (1) four steps or less, (0) more than four steps; technical skill needed by the operator: (1) laboratory experience not required, (0) need for laboratory experience; and total test execution time: (1) $30 \mathrm{~min}$ or less, (0) more than $30 \mathrm{~min}^{11}$

\section{Ethical considerations}

All plasma bag identifications were unlinked from the panel members prior to analysis, and no clinical and/or demographic data were obtained from the donors.

\section{Statistical analysis}

The sensitivity, specificity and accuracy of the RTs were calculated and expressed with a binomial CI of 95\%, using the MedCalc software (V.12.0.3.0, -Ostend/Belgium). Accuracy values were transformed into percentages and interpreted as excellent (90\%-100\%), good (80\%-90\%), fair (70\%-80\%), poor $(60 \%-70 \%) \%$ ) and weak $50 \%-60 \%)^{12}$

Kappa statistic was used to determine the concordance between the RTs for syphilis and the gold standard, and the values determined by Altman ${ }^{13}$ were used as reference. Poor concordance was given for Kappa values of between 0.00 and 0.20 ; fair $(0.21$ to 0.40$)$; moderate $(0.41-0.60)$; good $(0.61-0.80)$ and very good (0.81-1.00). ${ }^{13}$ The Statistical Package for Social Sciences (SPSS software, V.17.0) was used to determine the Kappa values.

\section{Results}

Table 1 shows that all the tests admit plasma, serum or whole blood samples as a matrix. All the tests have the same storage temperature range $\left(2^{\circ} \mathrm{C}-30^{\circ} \mathrm{C}\right)$, with the exception of the Rapid Check Syphilis test that must be stored at $5^{\circ} \mathrm{C}-30^{\circ} \mathrm{C}$.

One of the first steps of this evaluation was to establish a panel with samples duly classified in terms of their reactivity to the syphilis tests. This meant that it was subsequently possible to evaluate the RTs available in the Brazilian market. The initial strategy was to use bags of plasma obtained from blood donors as a sampling source. The size of each bag ensured that there was sufficient plasma to generate aliquots which, when correctly stored, can enable several evaluations to be carried out over the years.

After characterising the total of 493 samples, the first panel consisted of $127(25.8 \%)$ treponemal antibody-positive and 366 (74.2\%) antibody-negative specimens for syphilis. There was complete concordance between the results obtained with the three reference tests (FTA-ABS, chemiluminescence immunoassay and TPHA).

Among the evaluated tests, none of the seven RTs required samples to be inactivated. The sensitivity of the RTs evaluated 
Table 2 Sensitivity, specificity, accuracy and Kappa coefficient of the seven evaluated rapid tests

\begin{tabular}{|c|c|c|c|c|}
\hline Test & Sensitivity $\%(95 \% \mathrm{Cl})$ & Specificity $\%(95 \% \mathrm{Cl})$ & Accuracy \% $(95 \% \mathrm{Cl})$ & Kappa \\
\hline Imuno-Rapido Syphilis & $100(97.1$ to 100$)$ & 94.0 (91.0 to 96.2$)$ & 97.0 (95.1 to 98.3 ) & 0.89 \\
\hline Determine Syphilis & $100(97.1$ to 100$)$ & 91.5 (88.2 to 94.2 ) & 95.8 (93.6 to 97.4 ) & 0.85 \\
\hline Rapid-Check Syphilis & $99.2(95.7$ to 100$)$ & 99.7 (98.5 to 100$)$ & 99.5 (98.3 to 99.9) & 0.99 \\
\hline RT DPP Syphilis & 100 (97.1 to 100$)$ & 100 (99.0 to 100$)$ & 100 (99.3 to 100$)$ & 1 \\
\hline Syphilis Strip Test Bioeasy & 94.5 (89.0 to 97.8$)$ & 99.5 (98.0 to 100$)$ & 97.0 (95.0 to 98.3 ) & 0.95 \\
\hline RT ABON & $100(97.1$ to 100$)$ & 97.0 (94.7 to 98.5 ) & 98.5 (97.0 to 99.4$)$ & 0.94 \\
\hline Syphilis Test Biocon & 94.5 (89.0 to 97.8 ) & 97.8 (95.7 to 99.0 ) & 96.2 (94.1 to 97.7 ) & 0.92 \\
\hline
\end{tabular}

varied from $94.5 \%$ to $100 \%$, while their specificity ranged from $91.5 \%$ to $100 \%$ (table 2). It is important to note that the RT DPP Syphilis test correctly classified all the panel samples. With the exception of Determine Syphilis, which had a specificity of $91.5 \%$ (95\% CI 88.2 to 94.2$)$ and was therefore below the minimum $93 \%$ acceptable to the $\mathrm{MoH},{ }^{78}$ all the other tests had specificity above this value, ranging from $94 \%$ to $100 \%$. The accuracy of the tests ranged from $95.8 \%$ to $98.5 \%$.

The Kappa statistic was used to estimate the concordance level between the seven RTs evaluated and the results of the reference samples. Five (5/7) of the RTs presented an excellent Kappa index (0.92-1) and two others presented good concordance.

As for operational performance, all the RTs evaluated obtained a maximum score (five points) with the exception of the RT DPP Syphilis assay, which obtained 4 points due to the number of reagents used (two buffers) (table 3 ).

\section{DISCUSSION}

Syphilis diagnosis remains a challenge because the tests results need to be associated with the clinical history to be correctly interpreted. Countries with low prevalence of syphilis and well-distributed laboratory coverage do not recognise in the RTs a role to contribute for the improvement of syphilis diagnosis and have not found reasons to replace the regular syphilis laboratory tests. ${ }^{14}$ In addition, Food and Drug Administration has cleared only one syphilis RT in the USA. ${ }^{14}$ However, low-income countries or those that present specific characteristics have other challenges to face. For example, Brazil's huge continent-sized land area, with many remote and difficult-to-access places and often restricted internet access, often make it difficult to obtain the results of laboratory tests. As an integral part of the strategies for improving access to diagnosis and therefore care, since 2005, on-site rapid testing has enabled same-day test results and treatment as indicated. ${ }^{78}$ The centralised bulk procurement of RTs has produced substantial economies of scale. In 2014, 2015 and 2017, a total of 3960000,9298980 and 12037550 tests, respectively, were purchased in this way. In parallel to the introduction of RTs, Brazil implemented strategies to evaluate the quality of the tests as well as an external quality control assessment programme to cover the whole country and control the quality of the services delivered.

Obtaining reactive samples for syphilis is a fairly difficult task, especially in countries where syndromic treatment is widely available in the health services. In these circumstances, blood banks play an important role in providing syphilis plasma samples. From a legal standpoint, plasma bags with reagent results for any serological marker should be discarded as unfit for use in transfusions. They can also, for example, contain anticoagulants that lead to the formation of fibrin clumps that could interfere with certain syphilis test results (eg, VDRL). Furthermore, the plasma freeze-thaw process can facilitate the formation of precipitates. However, Castro et $a l^{6}$ demonstrated that plasma samples can be converted into serum that can be used for producing quality control panels. ${ }^{6}$ These authors showed that as a result of the plasma conversion process, there is virtually no loss of sample reactivity, since $85 \%$ of the samples retain the titre in the non-treponemal reagin plasma reaction (RPR) test. In the treponemal tests, the reactivity is maintained in $96 \%$ in FTA-ABS and in $89 \%$ in the TPHA. Since they are free of fibrin, plasma samples converted into serum can be kept frozen for long periods without precipitation and are therefore suitable for the manufacture of reference sera and quality control panels. The worldwide CDC and WHO Joint External Quality Testing Program for Syphilis tests uses plasma samples converted

Table 3 Evaluation of the operational performance of the test based on five characteristics of the RT

\begin{tabular}{|c|c|c|c|c|c|c|}
\hline \multirow[b]{2}{*}{ Assay } & \multicolumn{6}{|c|}{ Operational assay performance score } \\
\hline & No. of reagents & $\begin{array}{l}\text { Reagent storage } \\
\text { temperature }\end{array}$ & No. of steps & $\begin{array}{l}\text { Total execution time } \\
(\mathrm{hh}: \mathrm{mm})^{*}\end{array}$ & $\begin{array}{l}\text { Technical skills of } \\
\text { operator }\end{array}$ & Assay performance \\
\hline Determine Syphilis TP & $1(1)$ & $1\left(2-30^{\circ} \mathrm{C}\right)$ & $1(3)$ & $1(00: 16)$ & 1 & Good (5/5) \\
\hline Imuno-Rápido Syphilis & $1(1)$ & $1\left(2-30^{\circ} \mathrm{C}\right)$ & $1(3)$ & $1(00: 11)$ & 1 & Good (5/5) \\
\hline Rapid-Check Syphilis & $1(1)$ & $1\left(5-30^{\circ} \mathrm{C}\right)$ & $1(3)$ & $1(00: 11)$ & 1 & Good (5/5) \\
\hline SyphilisTest Biocon & $1(1)$ & $1\left(2-30^{\circ} \mathrm{C}\right)$ & $1(3)$ & $1(00: 11)$ & 1 & Good (5/5) \\
\hline Syphilis Strip Test Bioeasy & $1(1)$ & $1\left(2-30^{\circ} \mathrm{C}\right)$ & $1(3)$ & $1(00: 21)$ & 1 & Good (5/5) \\
\hline RT ABON & $1(1)$ & $1\left(2-30^{\circ} \mathrm{C}\right)$ & $1(3)$ & $1(00: 11)$ & 1 & Good (5/5) \\
\hline RT DPP Syphilis & $0(2)$ & $1\left(2-30^{\circ} \mathrm{C}\right)$ & $1(4)$ & $1(00: 16)$ & 1 & Good (4/5) \\
\hline
\end{tabular}

* Total execution time considered to be the time taken between the placement of the samples and the buffer (s) in the test device, plus the maximum time allowed for reading the result. The time taken to obtain the sample and prepare the work area for the test was not considered.

RT, rapid test. 
to serum. ${ }^{6}$ Since the defibrination methodology is very well described, this approach can be followed by other countries.

The panel used in the present study was made with plasma samples converted into serum. We used only true-positive and true-negative samples, since the objective was to verify the performance of the RTs as compared with the conventional tests routinely used in laboratories. The MoH's diagnosis access policy and the expansion of the diagnostic coverage for syphilis in pregnant women have adopted RTs as a screening test. In this respect, RTs are expected to perform as well as the treponemal tests used in the laboratory. The present study is the first to evaluate RTs for syphilis marketed in Brazil.

The sensitivity of the tests evaluated varied from $94.5 \%$ to $100 \%$ in relation to the panel and the specificity from $91.5 \%$ to $100 \%$. Imuno-Rapido Syphilis, Determine Syphilis, RT DPP Syphilis and RT ABON tests showed 100\% sensitivity with substantial variations in specificity values. The RT DPP Syphilis test showed $100 \%$ specificity, followed by RT ABON $(97.0 \%)$ and Imuno-Rapido Syphilis (94.0\%). Determine Syphilis $(91.5 \%)$ failed to reach the minimum values established by the $\mathrm{MoH}$ for this parameter (93.0\%). Rapid Check Syphilis (sensitivity $99.2 \%$ and specificity 99.7\%), Syphilis Strip Test Bioeasy $(94.5 \%$ and $99.5 \%)$ and Syphilis Test Biocon $(94.5 \%$ and $97.8 \%$ ) showed values of sensitivity and specificity of below $100 \%$ but nevertheless within the limits accepted by the $\mathrm{MoH}$. The kappa coefficients were between 0.89 and 1.0, indicating good and excellent concordance between the RTs and the gold standard.

Several studies report the variability of sensitivity values of the Determine Syphilis test. Results consistent with those found in the present study (100.0\%) were reported by Lien et $a l^{16}$ Hernández-Trejo $e$ t $a l^{17}$ and Siedner et al. ${ }^{18}$ Other authors reported low sensitivity values when using finger-prick, whole blood samples as $88.0 \%,{ }^{18} 91.8 \%,{ }^{19} 85.7 \%{ }^{20}$ and $97.3 \%{ }^{21} \mathrm{~A}$ multicentre clinic-based study performed by Mabey et $a^{22}$ also found low sensitivity in Tanzania (59.6\%), Brazil (88.5\%), China $(81.9 \%)$ and Haiti $(72.5 \%){ }^{22}$

Specificity values for the Determine Syphilis test similar to those found in this study were reported in the study by Brozan et $a l^{20}$ who found $90.9 \%$. Herring $e a^{23}$ in a multicentre study with archived samples, found specificity values ranging from $88.0 \%$ to $100.0 \% .^{23}$ Other authors have reported specificity values higher than those found in the present study, varying from $97.7 \%$ to $100.0 \%$. ${ }^{171822} 2425$ These findings suggest that important differences occur in the results obtained in different studies and raise questions about the variability of the test depending on the sample matrix used in the evaluation, as well as possible methodological modifications in the test, the influence of testing conditions (field or laboratory based) or lack of robustness of the test per se.

In the present study, Syphilis Strip Test Bioeasy showed values of $94.5 \%$ sensitivity and $99.5 \%$ specificity. The kappa coefficient $(0.95)$ indicates that this test is similar to the other members of this evaluation. Due to the change in the name of the test, the comparison in the literature was carried out with the test previously marketed in Brazil-SD Bioline Syphilis 3.0. It is possible, however, that in addition to the name change there may have been methodological modifications. Several studies have different specificity and sensitivity values with the SD Bioline Syphilis 3.0 test. Sensitivity values very close to those found in the present study were reported by Herring $e t a^{23}$ who found 95.0\%; Benzaken et al ${ }^{26}$ 90.2\%; Dlamini et al ${ }^{27} 90.1 \%$; Causer et $a l^{21} 87.8 \%$; Bocoum et $a l^{28} 90 \%$; and Mehra et al ${ }^{29}$ $92.9 \%$. As for specificity values, the same authors found $94.9 \%$,
$99.4 \%, 98.0 \%, 98.5 \%, 98.0 \%$ and $98.3 \%$, respectively. With the exception of the $94.9 \%$ obtained by Herring et $a^{23}$ the specificity values obtained by the other authors are in line with the $99.5 \%$ found in the present study. ${ }^{23}$

The RT DPP Syphilis test presented values of $100.0 \%$ sensitivity, specificity and kappa coefficient of 1 , presenting excellent performance in all the evaluated parameters. However, this was the only test that did not show maximum operational performance (table 3). The high performance of the test cannot be compared with other references since there are no studies on RT DPP Syphilis for a single marker on database platforms.

The tests Imuno-Rápido Syphilis (Wama Diagnostica), Rapid Check Syphilis (NDI-UFES), Syphilis Test Biocon (Biocon Diagnostics) and RT ABON (Abon, Hangzhou) have no validation studies available in database platforms, which rules out discussion here of their results, although they presented, respectively, sensitivity values $(100.0 \%, 99.2 \%, 94.5 \%$ and $100.0 \%)$, specificity $(94.0 \%, 99.7 \%-97.8 \%$ and $97.0 \%)$ and kappa coefficient $(0.89,0.99,0.92$ and 0.94$)$ above the minimum values established by the Brazilian $\mathrm{MoH}$.

The present study has the following limitations: (1) no clinical data were obtained, and therefore it was not possible to correlate the laboratory results with the clinical presentation, (2) the use of defibrinated plasma as a primary sample for the performance of reference tests and RTs, although it is not the matrix used in the public health services, is appropriate for the making up quality control panels; and (3) although the study was conducted in a laboratory environment with technical staff trained and experienced in performing RTs, it is expected that the results obtained in the health services are comparable with those obtained in the present evaluation. ${ }^{6}$ Despite these limitations, the results from this work can be extrapolated to the countries that use the same syphilis RTs.

Cost-effectiveness studies of different diagnostic strategies for syphilis indicate that an RT is cost-effective if the sensitivity is higher than $88 \%$. Failing that, the use of RPR is recommended. ${ }^{30}$

\section{CONCLUSION}

The RT DPP Syphilis, Imuno-Rápido Syphilis, Rapid-Check Syphilis, Syphilis Strip Test Bioeasy, RT ABON and Syphilis Test Biocon tests showed operational performance and sensitivity and specificity values as established in the Ministerial Directive for the diagnosis of syphilis in Brazil.

RTs for syphilis are an excellent alternative for use in the public health sphere because they enable diagnoses to reach populations that were previously excluded from access. Notwithstanding the benefits of RTs in the implementation of public health policies, the tests need to be carefully quality controlled, ideally, on a batch-to-batch basis.

\section{Key messages}

- The seven rapid tests evaluated in the study presented good operational performance.

- The professionals who performed the rapid tests considered them easy to use and interpret.

- Most tests reached sensitivity and specificity values as established in the Ministerial Directive for the diagnosis of syphilis in Brazil.

- Rapid tests for syphilis are an excellent alternative because they enable diagnosis to reach populations that were previously excluded from access. 


\section{Author affiliations}

'Department of Clinical Analysis, Health Sciences Center, Laboratory of Molecular Biology, Microbiology and Serology, Federal University of Santa Catarina, Florianopolis, Santa Catarina, Brazil

${ }^{2}$ Research Laboratory on HIVIAIDS, Center for Life Sciences and Health, University of Caxias do Sul, Rua Francisco Getúlio Vargas, Caxias do Sul, Brazil

${ }^{3}$ Faculty of Medicine, University of São Paulo, São Paulo, Brazil

${ }^{4}$ Department of Surveillance, Prevention and Control of Sexually Transmitted Infections, HIVIAIDS and Viral Hepatitis, Secretariat of Health Surveillance, Ministry of Health, Setor Administrativo Federal Sul, Brasília, Distrito Federal, Brazil

${ }^{5}$ Faculdade de Medicina, Faculdade de Ciências de Saúde, Programa de Pós Graduação em Saúde Coletiva, Universidade de Brasília, Campus Universitário Darcy Ribeiro, Brasília, Distrito Federal, Brazil

${ }^{6}$ Laboratory of Molecular Virology, Institute of Biology, Federal University of Rio de Janeiro, Avenida Carlos Chagas Filho, Rio de Janeiro, Brazil

Acknowledgements Our thanks to the Department of Surveillance, Prevention and Control of Sexually Transmitted Infections, HIVIAIDS and Viral Hepatitis team, to the Laboratory of Molecular Biology, Microbiology and Serology (LBMMS) of the Federal University of Santa Catarina staff and to John Penney and Leticia Muraro Wildner for the manuscript review.

Contributors Conception and design of the work: MLB and MF. Laboratory work and data analysis: RCMRdO, LG, AB, PCG and MLB. Drafting the work: MLB, LRdM, OdCFJ, AFNPCP and MF; critically revised the paper: OdCFJ, FM, MF and ASB. Responding to the editorial committee and the referees: MLB, RCMRdO, AB, PCG and AFNPCP. Final approval: ASB.

Funding This study was funded by the Fundo Nacional da Saúde (National Health Fund/Brazil).

Competing interests None declared.

Patient consent Detail has been removed from this case description/these case descriptions to ensure anonymity. The editors and reviewers have seen the detailed information available and are satisfied that the information backs up the case the authors are making.

Provenance and peer review Commissioned; externally peer reviewed.

Open Access This is an Open Access article distributed in accordance with the Creative Commons Attribution Non Commercial (CC BY-NC 4.0) license, which permits others to distribute, remix, adapt, build upon this work non-commercially, and license their derivative works on different terms, provided the original work is properly cited and the use is non-commercial. See: http://creativecommons.org/ licenses/by-nc/4.0/

(c) Article author(s) (or their employer(s) unless otherwise stated in the text of the article) 2017. All rights reserved. No commercial use is permitted unless otherwise expressly granted.

\section{REFERENCES}

1 World Health Organization. Global health sector strategy on sexually transmitted infections 2016-2021: towards ending STIS. Genebra:WHO Press, 2016.

2 Brazil. Boletim Epidemiológico Sífilis. In: Epidemiological Bulletin of Syphilis. BrasiliaDF: Ministry of Health, 2016.

3 World Health Organization. The Global elimination of congenital syphilis: rationale and strategy for action. Genebra: WHO Press, 2007.

4 Peeling RW, Ye H. Diagnostic tools for preventing and managing maternal and congenital syphilis: an overview. Bull World Health Organ 2004;82:439-46.

5 Pan American Health Organization. Guidance on syphilis testing. In: Latin America and the Caribbean: improving uptake, interpretation and quality of testing in different clinical settings. Washington, DC: PAHO, 2015.
6 Castro AR, Kikkert SE, Fears MB, et al. Defibrination of blood plasma for use in serological tests for syphilis. Clin Diagn Lab Immunol 2002;9:1376-8.

7 Brazil. Ministerial directive GM/MS n²012. Brasília-DF: M.s. office, Ministry of Health, 2016.

8 Brazil. Manual Técnico para Diagnóstico da Sífilis. In: Technical Guideline for the Diagnosis of Syphilis. Brasilia-DF: Ministry of Health, 2016.

9 Brazil. Ministerial Directive MS/SVS n²9. Brasília: M.d.S.S.d.V.e. Saúde, 2013.

10 Brazil. Manual técnico para o diagnóstico da infecção pelo HIV. In: Technical guideline for the diagnosis of HIV infection. Brasília, DF: Ministério da Saúde, Secretaria de Vigilância em Saúde, Departamento de DST, Aids e Hepatites Virais, 2013.

11 Ferreira Junior OC, Ferreira C, Riedel M, et al. Evaluation of rapid tests for anti-HIV detection in Brazil. AIDS 2005;19(Suppl 4):S70-S75.

12 Tape TG. Interpreting diagnostic tests: ROC curves: University of Nebraska Medical Center, 2013.

13 Altman DG. Pratical statistics for medical research. London: Chapman and Hall, 1991.

14 Peterman TA, Fakile YF. What is the use of rapid syphilis tests in the United States? Sex Transm Dis 2016:43:201-3.

15 Matthias J, Dwiggins P, Totten Y, et al. Notes from the field: evaluation of the sensitivity and specificity of a commercially available rapid syphilis test - Escambia County, Florida, 2016. MMWR Morb Mortal Wkly Rep 2016;65:1174-5.

16 Lien TX, Tien NT, Chanpong GF, et al. Evaluation of rapid diagnostic tests for the detection of human immunodeficiency virus types 1 and 2, hepatitis B surface antigen, and syphilis in Ho Chi Minh City, Vietnam. Am J Trop Med Hyg 2000;62:301-9.

17 Hernández-Trejo M, Hernández-Prado B, Uribe-Salas F, et al. [Maternal and congenital syphilis in two Mexican hospitals: evaluation of a rapid diagnostic test]. Rev Invest Clin 2006;58:119-25.

18 Siedner M, Zapitz V, Ishida M, et al. Performance of rapid syphilis tests in venous and fingerstick whole blood specimens. Sex Transm Dis 2004;31:557-60.

19 Tinajeros F, Grossman D, Richmond K, et al. Diagnostic accuracy of a point-of-care syphilis test when used among pregnant women in Bolivia. Sex Transm Infect 2006;82(Suppl 5):v17-v21.

20 Bronzan RN, Mwesigwa-Kayongo DC, Narkunas D, et al. On-site rapid antenatal syphilis screening with an immunochromatographic strip improves case detection and treatment in rural South African clinics. Sex Transm Dis 2007;34:S55-S60.

21 Causer LM, Kaldor JM, Fairley CK, et al. A laboratory-based evaluation of four rapid point-of-care tests for syphilis. PLoS One 2014;9:e91504.

22 Mabey D, Peeling RW, Ballard R, et al. Prospective, multi-centre clinic-based evaluation of four rapid diagnostic tests for syphilis. Sex Transm Infect 2006;82(Suppl 5):v13-v16.

23 Herring AJ, Ballard RC, Pope $\mathrm{V}$, et al. A multi-centre evaluation of nine rapid, point-ofcare syphilis tests using archived sera. Sex Transm Infect 2006;82(Suppl 5):v7-v12.

24 Gianino MM, Dal Conte I, Sciolè K, et al. Performance and costs of a rapid syphilis test in an urban population at high risk for sexually transmitted infections. J Prev Med Hyg 2007;48:118-22.

25 Villazón-Vargas N, Conde-Glez CJ, Juárez-Figueroa L, et al. [Evaluation of a rapid diagnostic test to assess the prevalence of maternal syphilis in Bolivia]. Rev Med Chil 2009:137:515-21.

26 Benzaken AS, Galbán García E, Sardinha JC, et al. Rapid tests for diagnosing syphilis: validation in an STD clinic in the Amazon Region, Brazil. Cad Saude Publica 2007;23(Suppl 3):S456-S457.

27 Dlamini NR, Phili R, Connolly C. Evaluation of rapid syphilis tests in KwaZulu-Natal. J Clin Lab Anal 2014;28:77-81.

28 Bocoum FY, Ouédraogo H, Tarnagda G, et al. Evaluation of the diagnostic performance and operational characteristics of four rapid immunochromatographic syphilis tests in Burkina Faso. Afr Health Sci 2015;15:360-7.

29 Mehra B, Bhattar S, Saxena S, et al. Evaluation of SD BIOLINE syphilis 3.0 for rapid diagnosis of syphilis: report from a regional sexually transmitted infection reference laboratory in North India. J Lab Physicians 2016;8:36-40.

30 Drancourt M, Michel-Lepage A, Boyer S, et al. The Point-of-care laboratory in clinical microbiology. Clin Microbiol Rev 2016;29:429-47. 